Colombo J, Koletzko B, Lampl M (eds): Recent Research in Nutrition and Growth. Nestlé Nutr Inst Workshop Ser, vol 89, pp 25-35, (DOI: 10.1159/000486490)

Nestlé Nutrition Institute, Switzerland/S. Karger AG., Basel, @ 2018

\title{
Critical Windows for the Programming Effects of Early-Life Nutrition on Skeletal Muscle Mass
}

\author{
Marta L. Fiorotto $\cdot$ Teresa A. Davis \\ Department of Pediatrics, United States Department of Agriculture/Agricultural Research Service \\ (USDA/ARS) Children's Nutrition Research Center, Baylor College of Medicine, Houston, TX, USA
}

\begin{abstract}
Skeletal myogenesis begins in the embryo with proliferation and differentiation of muscle progenitor cells that ultimately fuse to form multinucleated myofibers. After midgestation, muscle growth occurs through hypertrophy of these myofibers. The most rapid growth phase occurs in the perinatal period, resulting in the expansion of muscle mass from $25 \%$ of lean mass at birth to $40-45 \%$ at maturity. These 2 phases of muscle growth are regulated by distinct molecular mechanisms engaged by extracellular cues and intracellular signaling pathways and regulatory networks they activate. Nutrients influence muscle growth by both providing the necessary substrates and eliciting extracellular cues which regulate the signal transduction pathways that control the anabolic processes of the fibers. The uniquely large capacity of immature myofibers for hypertrophy is enabled by a heightened capacity and sensitivity of protein synthesis to feeding-induced changes in plasma insulin and amino acids, and the ability to expand their myonuclear population through proliferation of muscle precursor cells (satellite cells). With maturation, satellite cells become quiescent, limiting myonuclear accretion, and the capacity of the muscles for protein anabolism progressively diminishes. Therefore, the early developmental phases represent critical windows for muscle growth which, if disrupted, result in muscle mass deficits that are unlikely to be entirely recoverable.
\end{abstract}

๑) 2018 Nestlé Nutrition Institute, Switzerland/S. Karger AG, Basel

\section{Introduction}

Longitudinal studies of human cohorts, experimental animal models, and farm animal species have consistently observed that suboptimal nutrition during early life has long-term consequences on the skeletal musculature [1-3]. This out- 


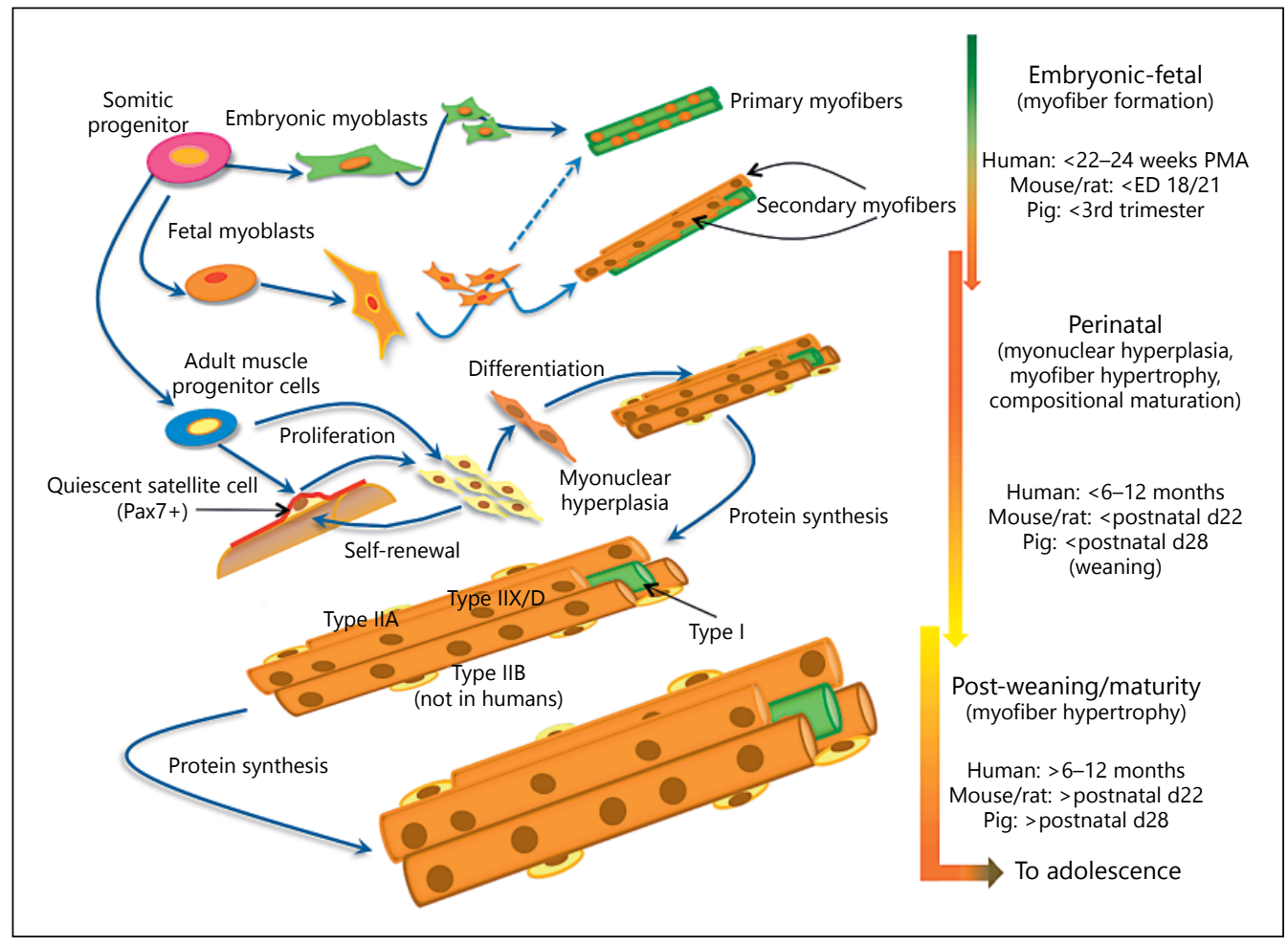

Fig. 1. Summary of major events that occur during myogenesis and muscle hypertrophy, and the corresponding developmental stages and chronological ages during which they occur in the human, rodents, and pigs [data compiled from references $5,7,10-15,19-21$, 23-24]. ED, embryonic day; PMA, postmenstrual age.

come appears to be critically dependent on the developmental age when the insult is experienced. Thus, understanding the specific cellular events responsible for muscle growth and development is essential in order to identify the windows of development when the organism is especially at risk for the reprogramming of skeletal muscle mass.

\section{Skeletal Muscle Development}

The development of the skeletal musculature can be divided into a series of temporally overlapping phases beginning with the formation of the somites of the early embryo and culminating in the fully differentiated tissue in the adult (Fig. 1) [4]. In the early stages, pluripotent mesodermal cells differentiate into muscle progenitor cells under the control of diffusible molecules. These under- 
go continued proliferation and migration from the somites into the various muscle beds where they fuse to form myotubes. Upon completion of this early phase, the organism has acquired its full complement of muscle fibers; in human and farm animals, this has occurred by approximately midgestation $[5,6]$, while in rodents it continues until birth [7]. Once myotubes have formed, a complex orchestrated change in gene expression drives the differentiation of the myofiber with the production of the complement of structural and functional proteins that confer the tissue its properties, i.e., contraction and force generation, ion and solute transport, and energy production. Concurrently, innervation and vascularization of the muscles support further specialization and refinement in functional and metabolic properties associated with the establishment of different fiber types [8]. A significant part of this developmental stage is completed within the early months of postnatal life in humans and in the early postnatal period in most mammalian species, as manifested in the advancing muscle-dependent functional abilities of the organisms.

The gain in muscle protein mass over this time is through growth in both girth and length of the myofibers. Its quantification can be assessed either from cadaver analysis, which provides an absolute measure of the whole-body amounts, or from the analysis of the cross-sectional areas of muscle fibers which provides an index of muscle size. At the end of fiber formation, approximately $20 \%$ of total body protein is in skeletal muscle; this increases to approximately $26 \%$ of body protein in the term fetus and to $40-45 \%$ by adulthood (more in males than females [reviewed in 9, 10]). These changes indicate that the accretion of skeletal muscle proteins is higher than that of other body protein pools and dominates the changes in lean body mass in the early years of life. Measurements of fiber cross-sectional areas are obtained more readily and provide greater detail on the pattern of muscle growth. These demonstrate that during the early stages of differentiation until the third trimester of pregnancy, fiber girth expands relatively slowly $[5,11]$. At the onset of myofiber differentiation, there is rapid hypertrophy that continues up to 2-6 months of age [10,11], after which growth decelerates and ceases by adolescence [10]. In rodents, a similar pattern occurs, with skeletal muscle protein mass comprising approximately $30 \%$ of body protein at birth, $45 \%$ by weaning, and then increasing more gradually to approximately $50 \%$ in adulthood [12].

\section{Delineation of Developmental Windows in Muscle Development}

Three broad windows can be defined for muscle growth (Fig. 1) and, although suboptimal nutrition retards muscle growth at any age, the specific cellular events that are affected at each stage differ. These differences are critical as they 
dictate whether the muscle can recuperate when adequate nutrition is restored. In the fetal phase when secondary muscle fibers are forming, maternal nutrient restriction results in a reduction in the number of fibers and, even though myofiber size may be recoverable, there is a permanent deficit in muscle mass because additional fibers are not generated once this window is closed $[6,13,14]$. This response is manifested only when there is very severe maternal nutrient restriction [14], and it may be attributable to concurrent maternal stress and nutrient deficits. Nonetheless, the reduction in fiber number in animals in which only nutrient flow to the fetus is disrupted, e.g., due to placental insufficiency, suggests that nutrient deficiency per se can impede myofiber formation [6, 13]. The exact mechanisms responsible for the impairment of this myogenesis step are uncertain. As indicated previously, there is little fiber hypertrophy at this time, and, thus, reduced myoblast proliferation, migration, and/or fusion as well as increased apoptosis are likely responsible. Whether this is also true for humans is uncertain, but there is no evidence to indicate that their response would differ.

The second phase spans the perinatal period to approximately weaning during which time the myonuclear number and protein mass of fibers increase rapidly. The acceleration in muscle hypertrophy over this period incurs a high nutrient cost which, if not met, leads to growth faltering. In species where this phase begins in late gestation, a compromised nutrient supply to the fetus leads to the diversion of blood flow and nutrients to spare the brain and organs at the cost of muscle growth [reviewed in 2]. Similarly, when the postnatal amino acid and energy requirements to support optimal growth are not met, skeletal muscle growth is preferentially blunted. Studies in experimental animal models [reviewed in 15] as well in human cohorts [1,3] provide strong evidence that deficits in muscle mass incurred during this phase cannot be recuperated entirely. During the third phase, attained once compositional and functional maturation are complete, muscle fiber hypertrophy continues at a slower rate, and any deficits incurred during this final window are recoverable.

The second window of muscle development, i.e., the perinatal phase, is of clinical significance because, if compromised, vital functions necessary for extrauterine survival, such as breathing, feeding, and locomotion, will be affected. For a newborn, breathing and feeding are essential for the transition to extrauterine life, and when their function is suboptimal, there are detrimental short- and long-term morbidities. In animals, the capacity for locomotor function is also critical for survival in the newborn, whereas in humans this function becomes important as the child begins to interact with its environment. In the long term, reduced muscle mass places the individual at in- 
Table 1. The postabsorptive and postprandial fractional rates of total protein synthesis, degradation, and accretion, and total RNA (as a measure of ribosomal abundance) in hind limb muscles of well-nourished rats from 6 to 70 days of age

\begin{tabular}{|c|c|c|c|c|c|}
\hline \multirow{2}{*}{$\begin{array}{l}\text { Postnatal } \\
\text { age } \\
\text { days }\end{array}$} & \multicolumn{2}{|c|}{ Synthesis, \%/day } & \multirow{2}{*}{$\begin{array}{c}\text { Degradation } \\
\% / \text { day }\end{array}$} & \multirow{2}{*}{$\begin{array}{l}\text { Accretion } \\
\% / \text { day }\end{array}$} & \multirow{2}{*}{$\begin{array}{c}\text { Total RNA }\left(C_{\mathrm{RNA}}\right) \\
\mathrm{mg} / \mathrm{g} \text { protein }\end{array}$} \\
\hline & $\begin{array}{l}\text { post- } \\
\text { absorptive }\end{array}$ & $\begin{array}{l}\text { post- } \\
\text { prandial }\end{array}$ & & & \\
\hline 6 & $16 \pm 4$ & $34 \pm 2$ & $15 \pm 1$ & $19 \pm 1$ & $38 \pm 1$ \\
\hline 10 & $12 \pm 3$ & $27 \pm 4$ & & & $32 \pm 3$ \\
\hline 15 & $13 \pm 3$ & $25 \pm 1$ & $12 \pm 1$ & $14 \pm 1$ & $21 \pm 1$ \\
\hline 22 & & $20 \pm 1$ & & & $14 \pm 1$ \\
\hline 28 & $11 \pm 2$ & $17 \pm 1$ & $9 \pm 1$ & $8 \pm 1$ & $9 \pm 0.1$ \\
\hline 43 & & $6 \pm 1$ & & & $8 \pm 0.1$ \\
\hline 70 & $3 \pm 1$ & $4 \pm 1$ & $4 \pm 1$ & $0 \pm 1$ & $5 \pm 0.1$ \\
\hline
\end{tabular}

Values are means $\pm \mathrm{SE} ; n=6-10$ per age. $C_{\mathrm{RNA}}$, translational capacity. Data are compiled from Davis et al. [17] and unpublished data.

creased risk of developing obesity, insulin resistance, and early-onset sarcopenia, and reduces their productivity, quality of life, and longevity $[2,3,16]$. Thus, it is essential that we understand the mechanisms that confer anabolic potential to the newly formed muscle so that we have a thorough understanding of how the nutritional management of neonates during this window of development can be optimized to ensure that muscle growth is not compromised.

\section{Mechanisms Driving Perinatal Muscle Hypertrophy}

The acceleration in fiber hypertrophy in the perinatal period is the product of muscle protein accretion supported by the addition of myonuclei. Net protein deposition occurs when rates of protein synthesis are higher than degradation (Table 1). The primary factors that regulate protein synthesis are the maximum capacity for translation, which is determined by ribosomal abundance, and translational efficiency, which is dependent on the rate of translation initiation. In the immature muscle, protein synthesis is higher than at any other time in life and is the main driver of muscle protein accretion [12]. These high rates of protein synthesis, however, are manifested only in the fed state (Table 1) [17]. As muscle matures, postprandial protein synthesis rates diminish, and protein deposition falls in parallel. The concurrent decreases in postabsorptive synthesis rates and protein degradation are modest and make a small contribution to the 
developmental decline in protein deposition (Table 1). The high maximal capacity for protein synthesis is dictated by ribosomal abundance (reflected in total muscle RNA content). In the hind limb muscles of the newborn rat, values decrease by $70 \%$ from birth to maturity with minor changes in translational efficiency (Table 1).

The second property of the immature muscle is its ability to accelerate protein synthesis following a meal. The principal mediator of this response is the surge in plasma insulin and amino acids, particularly the branched-chain amino acid, leucine [12]. This rapid rise in insulin and amino acids independently stimulates translation initiation via the activation of the phosphoinositide 3-kinase/ Akt-mechanistic target of rapamycin complex 1 (mTORC1) and the leucinemTORC1 signaling pathways, respectively [12]. In contrast, when nutrients are delivered continuously, these signaling pathways and protein synthesis are muted as a result of low, constant levels of insulin and amino acids [18]. This response of the immature muscle to meal feeding emphasizes the importance of the postprandial surge in insulin and amino acids for eliciting the stimulation in protein synthesis during this anabolic window of muscle development. In more mature muscle, similar increases in plasma insulin and amino acids in response to a meal do not elicit the same magnitude of change in protein synthesis (Table 1). This capacity of the muscle to accelerate protein synthesis when nutrients are available channels amino acids towards anabolic processes so that they are not available for oxidation, and thereby promotes greater efficiency in the use of dietary amino acids for growth. Therefore, in the immature animal, feeding becomes a prime regulator of muscle growth. Moreover, as skeletal muscle is the largest and most rapidly expanding protein pool in the body at this time, it is a primary determinant of amino acid requirements.

The rapid hypertrophy of the myofibers also requires an increase in myonuclear abundance. Myonuclei, however, are postmitotic, and an increase in their numbers following fiber differentiation depends on the activity of a muscle progenitor cell population known as satellite cells that can both selfrenew or terminally differentiate into myonuclei (Fig. 1). Their numbers are most abundant soon after myofibers form, and their proliferation and differentiation contribute to the rapid rise in myonuclei at this stage $[19,20]$. As terminal maturation progresses, satellite cell numbers decrease, and they become quiescent with myonuclear accretion reaching a nadir at approximately 3 weeks of age in the hind limb muscle of mice [20] and within 2-4 months of age in human abdominal muscles [21]. Studies in which satellite cell-proliferative capacity was impaired through genetic manipulations also demonstrate an absolute requirement for satellite cell proliferation for fiber hypertrophy during the suckling period in mice [22]. During the third phase, there 

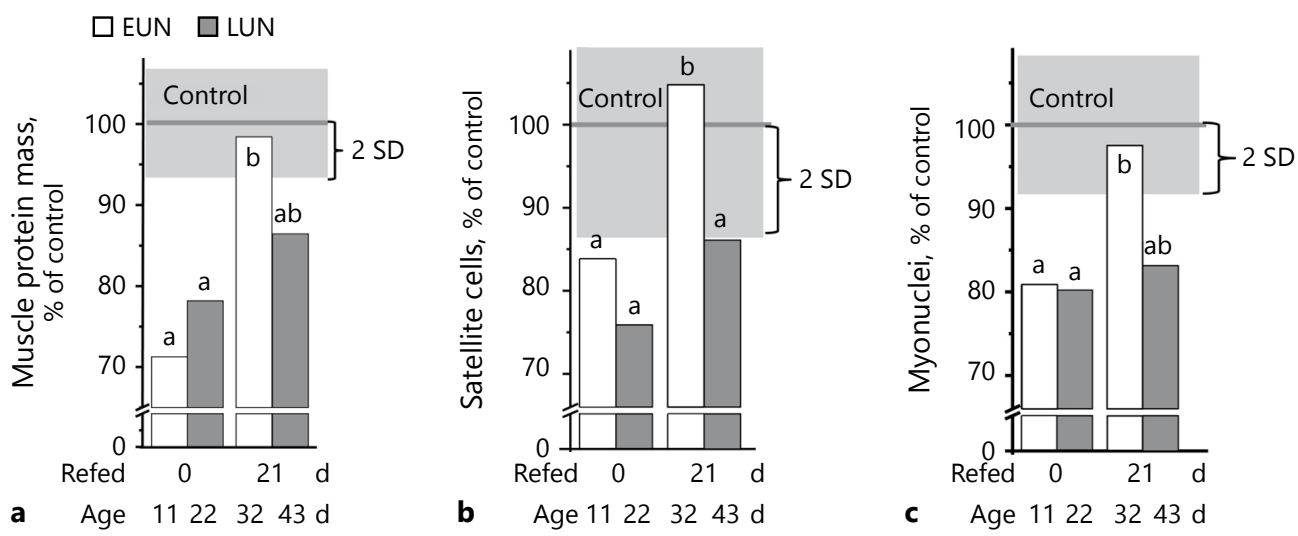

Fig. 2. Responses of muscle protein mass per millimeter bone length (a), satellite cell numbers (b), and myonuclear abundance per fiber profile (c) in hind limb muscle of mice that had been undernourished from birth to 11 days (d) of age (EUN) or from 11 to 22 days of age (LUN), and after 22 days of nutritional rehabilitation. Values are expressed as percentages of age-matched controls, shown as $100 \pm 2 \%$ SD (shaded area). $n=7-9$ mice/ group. ${ }^{\mathrm{a}} p<0.05$ vs. age-matched control; ${ }^{\mathrm{b}} p<0.05$ vs. undernourished state. Data are from Fiorotto et al. [15] and unpublished data.

is little further addition of myonuclei, but continued protein deposition increases fiber hypertrophy and is manifested by the enlargement of myonuclear domain size.

\section{Effect of Developmental Age on the Capacity to Recuperate Muscle Mass}

We have assessed the short- and long-term consequences of suboptimal nutrition on the processes that regulate skeletal muscle growth during the perinatal period in experimental models. To identify the specific importance of the timing of the nutritional insult on the recuperative capacity of muscles, we have used a mouse model in which offspring were undernourished either from birth to 11 days of age (early undernutrition, EUN) or from 11 to 22 days of age (late undernutrition, LUN) [15]. To achieve the restriction, dams were fed a restricted protein diet ad libitum throughout lactation which limits milk production with little effect on milk macronutrient composition. Thus, pups essentially experience a global nutrient deficit. Following the period of restriction, nutritional rehabilitation was instituted either by suckling pups on well-nourished dams (EUN) or by 
providing them a control diet (LUN). The EUN group, therefore, experienced a growth deficit in a highly anabolic period, but was recovered before their muscles attained maturity. The LUN group was undernourished for the same length of time but recovered at a more advanced stage of maturity when there is little myonuclear proliferation, and muscle hypertrophy is primarily due to protein accretion. We observed that EUN resulted in a greater deficit in muscle protein than LUN (Fig. 2a). With refeeding, the EUN pups mounted a robust anabolic response and muscle protein mass was recovered (Fig. 2a). However, although the LUN pups exhibited some initial catchup growth, this was not sustained, and a deficit in muscle protein mass persisted into old age [15]. To identify the mechanisms responsible for the disparate responses, we assessed the protein-synthetic response to refeeding. We observed that with refeeding EUN pups accelerated postprandial protein synthesis above control levels and sustained this enhanced response until muscle protein mass was restored to the level of well-nourished controls. The LUN offspring exhibited a blunted response that was insufficient to restore muscle mass. Evaluation of the regulatory processes revealed no differences in the efficiency of the signaling pathways that stimulate translation initiation. In contrast, the more immature muscle of EUN offspring increased ribosomal abundance upon refeeding, a response that did not occur in the older LUN group. Further evaluation established that the increased ribosomal abundance was associated with increased expression of the nucleolar transcription factor, UBF, in EUN but not LUN progeny. This factor plays a critical function in regulating rRNA transcription, the limiting factor for ribosomal production.

Like the response in protein accretion, accumulation of myonuclei is also sensitive to nutrient supply during the early life phase of muscle growth. Suboptimal nutrition invariably has been associated with a reduction in myonuclei [23], and reduced satellite cell numbers and proliferative activity have been observed in various animal models of intrauterine and neonatal nutrient restriction [24] and in muscles of undernourished children [25]. In newborn pigs, within less than 48 $\mathrm{h}$ of feeding a diet providing adequate energy but only $50 \%$ of protein requirements, satellite cell proliferation had decreased to approximately $60 \%$ of control levels [26]. However, it was uncertain whether satellite cell proliferation could recover to restore myonuclear numbers and support catchup growth when the nutritional insult extends into the postnatal period. Thus, we used the previously described nutritional paradigm and determined that in both EUN and LUN groups there was a similar deficit of approximately $20 \%$ in satellite cells (Fig. 2b) and myonuclei (Fig. 2c) at the end of the period of undernutrition. Following 3 weeks of refeeding, satellite cell and myonuclear abundance were restored in the EUN group, but there was no restoration of satellite cell numbers and only a modest improvement in myonuclei in the LUN group. 


\section{Regulation of the Perinatal Window Aperture}

The fundamental mechanism responsible for orchestrating these developmental windows is unclear. Extensive research has implicated significant roles for the IGF and TGF- $\beta$ families of growth factors in the determination of skeletal muscle mass - the former as a positive regulator and the latter as an inhibitor of muscle growth [27]. IGF-I and -II signaling via the IGF-I receptor (1R) plays a significant role in the regulation of skeletal muscle growth by stimulating muscle protein anabolism and promoting satellite cell proliferation and myonuclear accretion [28]. The importance of the IGFs and the IGF-1R for in vivo muscle growth in early life was demonstrated by the blunted growth that occurred with globally disrupted expression of IGF-I, IGF-II, or IGF-1R [28]. However, it is the locally produced IGFs acting in an autocrine/paracrine mode that are considered to have the predominant influence on muscle growth [29]. This is further supported by the hypertrophic response observed in transgenic muscle-specific IGF-I mouse models [29]. It is highly pertinent, therefore, that the expression rates of IGF-I, IGF-II, and IGF-1R in muscles are high following differentiation and decrease during the maturation phase, attaining a nadir at approximately the same time that myonuclear accretion abates [15]. In contrast, myostatin acts through signaling pathways to blunt the proliferation and differentiation of committed myoblasts, while also inhibiting protein anabolism [27]. The expression of myostatin changes in a reciprocal manner to the IGFs in the developing muscle [30], and it is upregulated in catabolic states associated with muscle wasting. Recently, using genetic mouse models, we demonstrated that in conditions in which myostatin expression is ablated, while IGF-I is overexpressed in muscle, there is a synergistic effect on muscle hypertrophy [27]. This is not dissimilar to the condition of the immature muscle, and, although the significance of such a mechanism in the delineation of the perinatal window of muscle development requires further validation, it is consistent with other examples in which the progress from one step of development to the next is regulated by changes in the balance between positive and negative regulators [31,32].

\section{Translational Considerations}

The preponderance of evidence demonstrates that inadequate nutrient intake during early life can program low adult muscle mass. Moreover, the developmental age when nutritional rehabilitation can be restored following an episode of poor nutrition may be as critical as the duration or severity of the nutrient restriction. Identification of the factors responsible for this age-dependent re- 
sponse, as well as clarification of the role of specific nutrients in modulating the responses, will facilitate translation of the findings to human infants. The results emphasize the importance of considering developmental age in the nutritional management of infants undergoing catchup growth and those for whom standard feeding is precluded.

\section{Acknowledgments}

These studies were supported by grants from USDA CRIS 6250-51000-043, 051, and 060, NIH AR46308, NIH HD085573, NIH HD072891, USDA NIFA 2013-67015-20438, and Abbott Nutrition. This work is a publication of the Department of Pediatrics, USDA/ ARS Children's Nutrition Research Center, Baylor College of Medicine. The contents of this publication do not necessarily reflect the views or politics of USDA, nor does the mention of trade names, commercial products, or organizations imply endorsements by the US Government.

\section{Disclosure Statement}

The authors declare no conflicts of interest.

\section{References}

1 Sayer AA, Cooper C: Fetal programming of body composition and musculoskeletal development. Early Hum Dev 2005;81:735-744.

2 Brown LD: Endocrine regulation of fetal skeletal muscle growth: impact on future metabolic health. J Endocrinol 2014;221:R13-R29.

3 Kulkarni B, Hills AP, Byrne NM: Nutritional influences over the life course on lean body mass of individuals in developing countries. Nutr Rev 2014;72:190-204.

4 Sambasivan R, Tajbakhsh S: Skeletal muscle stem cell birth and properties. Semin Cell Dev Biol 2007; 18:870-882.

5 Stickland NC: Muscle development in the human fetus as exemplified by m.sartorius: a quantitative study. J Anat 1981;132:557-579.

6 Du M, Tong J, Zhao J, et al: Fetal programming of skeletal muscle development in ruminant animals. J Anim Sci 2010;88:E51E60.
7 Ontell M, Kozeka K: Organogenesis of the mouse extensor digitorum longus muscle: a quantitative study. Am J Anat 1984;171:149161.

8 Schiaffino S, Reggiani C: Fiber types in mammalian skeletal muscles. Physiol Rev 2011;91: 1447-1531.

9 Forbes GB: Human Body Composition: Growth, Aging, Nutrition and Activity. New York, Springer, 1987.

10 Malina R: Growth of muscle tissue and muscle mass; in Falkner F, Tanner JM (eds): Human Growth. New York, Plenum Press, 1978, vol 2, pp 273-294.

11 Colling-Saltin A-S: Enzyme histochemistry on skeletal muscle of the human foetus. J Neurol Sci 1978;39:169-185.

12 Davis TA, Fiorotto ML: Regulation of muscle growth in neonates. Curr Opin Clin Nutr Metab Care 2009;12:78-85.

13 Wigmore PM, Stickland NC: Muscle development in large and small pig fetuses. J Anat 1983;137:235-245. 
14 Wilson SJ, Ross JJ, Harris AJ: A critical period for formation of secondary myotubes defined by prenatal undernourishment in rats. Development 1988;102:815-821.

15 Fiorotto ML, Davis TA, Sosa HA, et al: Ribosome abundance regulates the recovery of skeletal muscle protein mass upon recuperation from postnatal undernutrition in mice. J Physiol 2014;592:5269-5286.

16 Wolfe RR: The underappreciated role of muscle in health and disease. Am J Clin Nutr 2006;84:475-482.

17 Davis TA, Fiorotto ML, Nguyen HV, et al: Response of muscle protein synthesis to fasting in suckling and weaned rats. Am J Physiol 1991;261:R1373-R1380.

18 Davis TA, Fiorotto ML, Suryawan A: Bolus vs continuous feeding to optimize anabolism in neonates. Curr Opin Clin Nutr Metab Care 2015;18:102-108.

19 Allbrook DB, Han MF, Hellmuth AE: Population of muscle satellite cells in relation to age and mitotic activity. Pathology 1971;3: 233-243.

20 White RB, Bierinx AS, Gnocchi VF, et al: Dynamics of muscle fibre growth during postnatal mouse development. BMC Dev Biol 2010;10:21.

21 Delhaas T, Van der Meer SF, Schaart G, et al: Steep increase in myonuclear domain size during infancy. Anat Rec (Hoboken) 2013; 296:192-197.

22 Lepper C, Conway SJ, Fan CM: Adult satellite cells and embryonic muscle progenitors have distinct genetic requirements. Nature 2009; 460:627-631.

23 Glore SR, Layman DK: Cellular development of skeletal muscle during early periods of nutritional restriction and subsequent rehabilitation. Pediatr Res 1983;17:602-605.
24 Beermann DH, Hood LF, Liboff M: Satellite cell and myonuclei populations in rat soleus and extensor digitorum longus muscles after maternal nutritional deprivation and realimentation. J Anim Sci 1983;57:1618-1625.

25 Hansen-Smith FM, Picou D, Golden MH: Muscle satellite cells in malnourished and nutritionally rehabilitated children. J Neurol Sci 1979;41:207-221.

26 Kao M, Columbus DA, Suryawan A, et al: Enteral $\beta$-hydroxy- $\beta$-methylbutyrate supplementation increases protein synthesis in skeletal muscle of neonatal pigs. Am J Physiol Endocrinol Metab 2016;310:E1072-E1084.

27 Hennebry A, Oldham JM, Shavlakadze T, et al: IGF1 stimulates greater muscle hypertrophy in the absence of myostatin in male mice. J Endocrinol 2017;234:187-200.

28 Yakar S, Adamo ML: Insulin-like growth factor 1 physiology: lessons from mouse models. Endocrinol Metab Clin North Am 2012;41: 231-247.

29 Shavlakadze T, Winn N, Rosenthal N, et al: Reconciling data from transgenic mice that overexpress IGF-I specifically in skeletal muscle. Growth Horm IGF Res 2005;15:4-18.

30 Suryawan A, Frank JW, Nguyen HV, et al: Expression of the TGF- $\beta$ family of ligands is developmentally regulated in skeletal muscle of neonatal rats. Pediatr Res 2006;59:175179.

31 Ishibashi M: Molecular mechanisms for morphogenesis of the central nervous system in mammals. Anat Sci Int 2004;79:226-234.

32 Aulehla A, Pourquie O: Signaling gradients during paraxial mesoderm development. Cold Spring Harb Perspect Biol 2010; 2:a000869. 
